\title{
Adenoid cystic carcinoma of the breast: case report
}

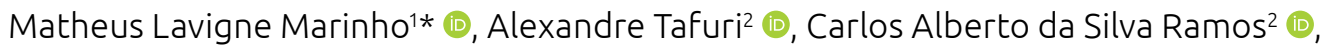 \\ Antônio Alexandre Lisbôa Ladeia ${ }^{2}$ [0, Luciana de Carvalho Azevedo 3 (1)
}

\section{ABSTRACT}

Adenoid cystic carcinoma (AdCC) of the breast is an uncommon invasive lobular neoplasm whose morphology is similar to the homonymous tumor of salivary glands and with a peculiar behavior toward the "triple-negative" (TN) profile. Tumors belonging to this family do not immunohistochemically express three of the main prognostic biomarkers and tend to show a more aggressive behavior. However, this rare histological pattern of breast cancer is generally associated with good prognosis. In this study, the authors describe the case of a 49-year-old woman diagnosed with this rare malignant tumor and who underwent breast-conserving surgery. Recent studies have aimed to understand the genes, genetic alterations, and etiological aspects related to the still obscure etiopathogenesis of AdCC. Thus, morphological and molecular aspects relevant to AdCC and reported in the literature will be discussed.

KEYWORDS: adenoid cystic carcinoma; breast neoplasms; triple-negative breast neoplasms.

\section{INTRODUCTION}

Currently, breast cancer stands out in prevalence among women, associated with increasing longevity, new lifestyle habits, and early menopause $\mathrm{e}^{1}$. Accurate anatomopathological diagnosis of tumors is essential to adopt an adequate and effective therapeutic approach, enabling satisfactory patients' survival ${ }^{1,2}$. Among the different histological types of breast cancer, adenoid cystic carcinoma (AdCC) stands out for being uncommon and presenting peculiar morphological and immunohistochemical characteristics, which provide a paradoxically favorable prognosis ${ }^{2}$. Due to the rare incidence, many cases of AdCC are not properly recognized or recorded in epidemiological databases, hindering the elucidation of AdCC etiopathogenic correlations ${ }^{2,3}$. In a recent publication on breast neoplasms, the World Health Organization (WHO) histologically subclassifies AdCC into classic, solid-basaloid, or with high-grade transformation ${ }^{2}$. These definitions are essentially based on architectural, cytological, and immunohistochemical characteristics, but they can also be objectified by genomic profiling ${ }^{2,4,5}$. Genomic studies performed by in situ hybridization (FISH) or by polymerase chain reaction (PCR) have gained prominence in the characterization and understanding of the AdCC etiopathogenesis ${ }^{4}$. The present case report addresses the diagnosis of an uncommon malignant breast tumor compatible with classic AdCC of the breast after histological and immunohistochemical evaluation.

\section{CASE REPORT}

A 49-year-old woman sought a mastology service due to the presence of a mass in the left breast. Despite apparently normal nipples and breasts, absence of bulging or skin retraction, a medium-radiodensity nodule with partially defined contours was observed at 2 o'clock in the left upper lateral quadrant, measuring $1.1 \mathrm{~cm}$ (Figure 1A). As no suspicious microcalcifications and alterations in the lymph nodes of the left axillary region were evidenced, it was classified as BI-RADS 0 . According to ultrasonography exam, there was a lesion suggestive of a BI-RADS 4 solid nodule, described as a nodular image, solid, rounded, hypoechoic, heterogeneous, with regular contours, with no flow capture on Doppler, $30 \mathrm{~mm}$ from the nipple, $12.8 \mathrm{~mm}$ from the skin, and measuring $9.6 \times 8.1 \mathrm{~mm}$ (Figures $1 \mathrm{~B}$ and $1 \mathrm{C}$ ). All identified lymph nodes were echographically normal.

For anatomopathological analysis, core biopsy products and a sectionectomy surgical specimen of the upper lateral quadrant of the left breast were obtained, in addition to a biopsy of the patient's sentinel lymph node. According to macroscopic inspection, the tumor was a white nodule, measuring $2.5 \mathrm{~cm}$, located deep to the breast (Figure 1D), whereas the lymph nodes, sentinel or non-sentinel, were soft to the cut with light-brown color and approximate size of $1.0 \mathrm{~cm}$. Histological analysis showed a neoplasm consisting of epithelial cells in a tubular and cribriform pattern, with few solid elements [score 1], similarly to the salivary gland tumor, diffusely infiltrating the breast parenchyma and adipose tissue. In addition, round and elongated

\footnotetext{
${ }^{1}$ Faculdade de Medicina, Universidade Federal de Minas Gerais - Belo Horizonte (MG), Brazil.

2Laboratório Tafuri de Patologia - Belo Horizonte (MG), Brazil.

${ }^{3}$ Hospital Alberto Cavalcanti, Rede Fundação Hospitalar do Estado de Minas Gerais - Belo Horizonte (MG), Brazil.

*Corresponding author: matheuslavignepato@gmail.com

Conflict of interests: nothing to declare.

Received on: 02/05/2021. Accepted on: 06/09/2021
} 
cells with moderate nuclear atypia [score 2], low mitotic index [score 1] as well as substance in the gland lumens (sometimes basophilic, sometimes eosinophilic) were verified. In special Alcian Blue staining, myxoid materials were observed in the basal lamina and gland content, confirming mucopolysaccharide composition (Figure 2).

The immunohistochemical study showed negativity for estrogen, progesterone, and HER2 receptors, dual cell population, epithelial and myoepithelial, as well as positivity for Ki-67 and CD117 (c-KIT) (Table 1).

Considering all the characteristics of the neoplasm, the following diagnosis was concluded: adenoid cystic carcinoma of the breast, with the following pathological staging: $p T 2 p N O p M X$. Taking into account the known favorable prognosis of this carcinoma and the absence of metastases, the propaedeutic and curative approach of sectionectomy dispensed with chemotherapy or radiotherapy. Furthermore, regular mastology follow-up was adopted with the patient for active surveillance of tumor recurrence.
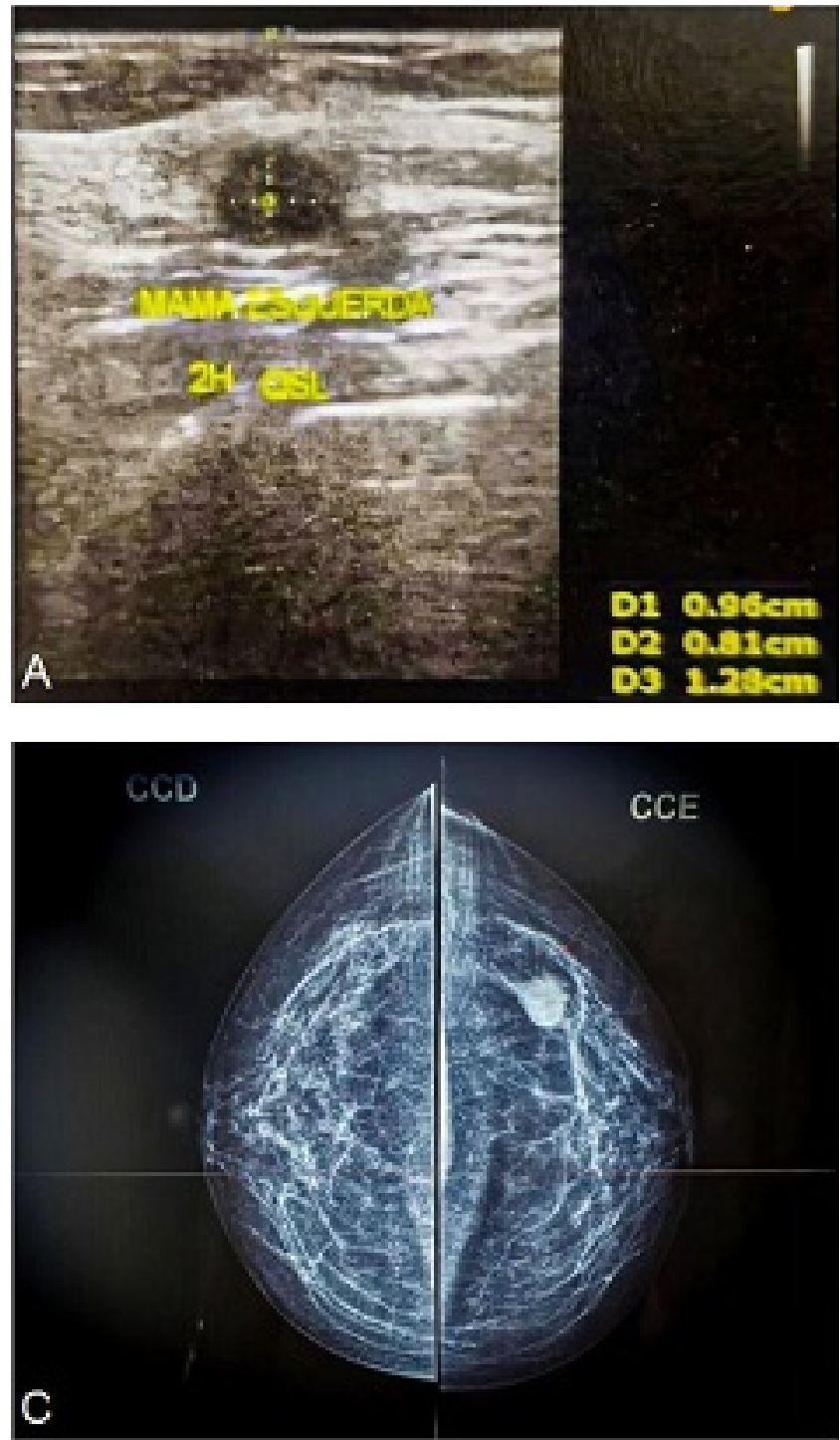

\section{DISCUSSION}

Adenoid cystic carcinoma of the breast (AdCC), a rare and important variant of invasive carcinoma, is worthy of attention of pathologists who routinely deal with breast biopsies ${ }^{6,7}$. Ghabach et al. estimated an age-adjusted incidence rate of 0.92 for every 1 million people/year, predominantly verified in postmenopausal women with a median age of 60 years $^{8}$. This epidemiological finding is corroborated by studies showing an incidence rate ranging between $0.1 \%$ and $3.5 \%$ among all breast carcinomas and age ranging between 33 and 74 years ${ }^{2,4,6,8,9}$. With a histological aspect resembling the homonymous tumor of salivary glands, in the breast, for classic types of AdCC, it requires a differential diagnosis with collagenous spherulosis, intraductal carcinoma with cribriform pattern ${ }^{5,6,10}$. As for the solid variant of AdCC, it requires differentiating it from neuroendocrine carcinoma, solid papillary carcinoma, metaplastic carcinoma, and malignant lymphoma ${ }^{4,6,9,11}$. Although the etiopathogenic relationship has not yet been confirmed, some authors suggest an association of

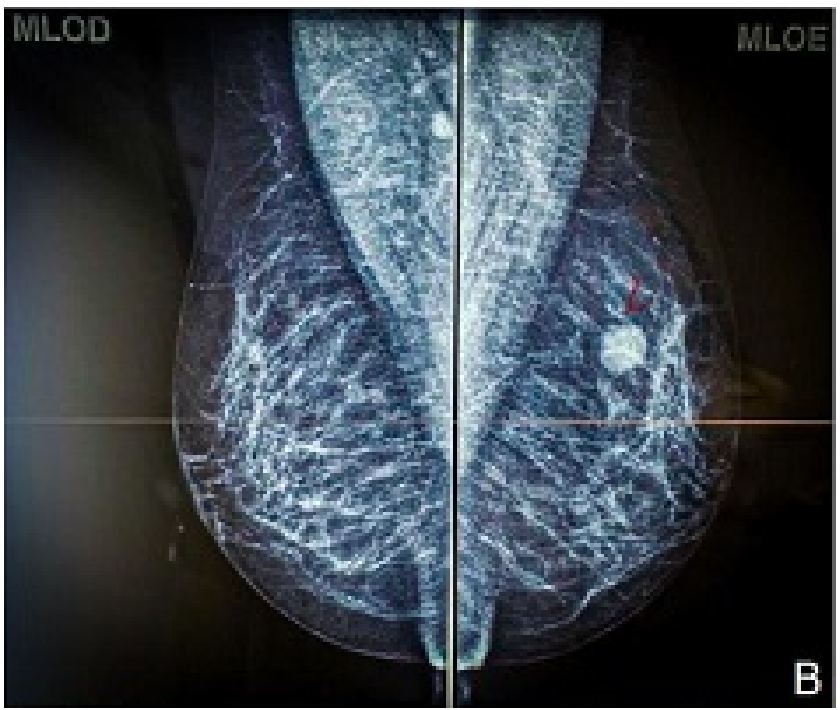

Figure 1. Macroscopic aspects of adenoid cystic carcinoma of the breast in imaging and anatomopathological tests. 
AdCC with benign lesions such as microglandular adenosis, tubular adenosis, adenomyoepithelioma and fibroadenoma $a^{2,4,5,12}$.

The tumor is histologically composed of a dual cell population (epithelial and myoepithelial), with a triple-negative molecular profile for estrogen receptor (ER), progesterone receptor (PR), and human

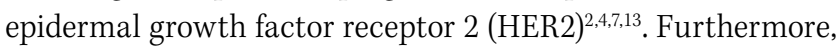
it presents basophilic secretions, formed by materials from the basal membrane, in the pseudoglandular lumens, which are better observed in the special Alcian Blue or PAS (Periodic acid-reactive Schiff) stains ${ }^{2,8,9,12}$. Other findings that support the AdCC hypothesis are potential immunohistochemical markers, such as CD117 and $\mathrm{Ki}-67$, as well as the evaluation of MYB-NIFB gene fusion or mutated genes BRAF, FGFR1/2, ERBB2, and NOTCH1, through molecular cytogenetic techniques as PCR or $\mathrm{FISH}^{2,4,6,9,12}$.

Among these mutations, the activation of NOTCH1, simultaneously considered oncogene and tumor suppressor gene, is identified in solid and triple-negative (TN) tumors, such as AdCC, influencing resistance to chemotherapy drugs ${ }^{2,14}$. In vitro and in vivo studies performed by Stoeck et al. showed that, unlike NOTCH2 and HES4 biomarkers, the increasing expression of NOTCH1 induces sensitivity to the gamma-secretase inhibitor MRK-003, as monotherapy or combined with the antineoplastic drug Paclitaxel ${ }^{14}$ The transcription product of this mutated gene is significantly

Table 1. Immunohistochemical profile of the tumor based on the sectionectomy product.

\begin{tabular}{|c|c|c|}
\hline Antibody & Clone & $\begin{array}{c}\text { Result } \\
\text { (neoplastic cells, \%) }\end{array}$ \\
\hline Estrogen Receptor & ER1 & Negative (0) \\
\hline Progesterone Receptor & PgR636 & Negative (0) \\
\hline HER2 oncogene product & SP3 & Negative (score 0 ) \\
\hline Ki-67: Cell proliferation antigen & MIB1 & Positive (15) \\
\hline $\begin{array}{l}\text { Calponin (muscle and } \\
\text { myoepithelial cells) }\end{array}$ & Calp & Focally positive \\
\hline $\begin{array}{l}\text { Tumor Protein p63 (squamous/ } \\
\text { transitional epithelium; } \\
\text { myoepithelial cells) }\end{array}$ & DAK-p63 & $\begin{array}{c}\text { Positive } \\
\text { (myoepithelial cells) }\end{array}$ \\
\hline CD117 - KIT gene product & YR145 & Positive \\
\hline
\end{tabular}

KIT: Proto-Oncogene Receptor Tyrosine Kinase; HER2: Human epidermal growth factor receptor 2 .
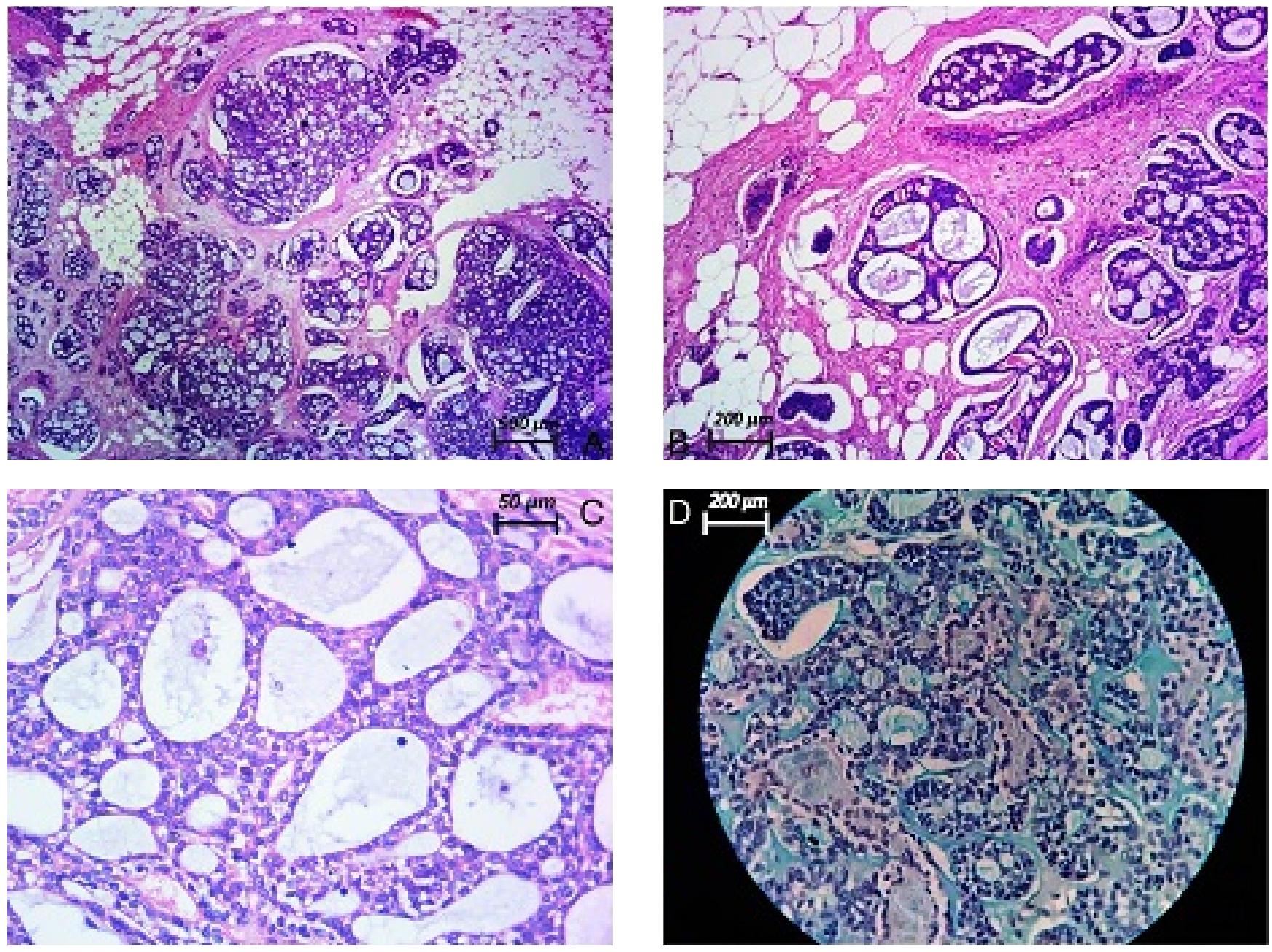

Figure 2. Microscopic aspects of adenoid cystic carcinoma of the breast. 
higher in basal-like and mesenchymal TN tumors ${ }^{13,14}$. These basallike tumors are subclassified into types 1 and 2, according to genetic expression, influencing sensitivity to chemotherapeutics $^{14,15}$. Despite sharing morphological characteristics with solidbasaloid AdCC, the differential diagnosis is enabled by investigating the tumor extension and identifying typical areas of $\mathrm{AdCC}^{2}$.

On mammography, thelesion, whose size varies between 1 and 140 $\mathrm{mm}$, is observed as alobulated or irregularmass, sometimes cystic, which mayhave defined borders; on ultrasound, it is solid and hypoechoic, or a heterogeneous mass ${ }^{6,9,16}$. According to guidelines from the American Society of Clinical Oncology/College of American Pathologists, breast tumors suspected of malignancy should be biopsied by core biopsy for immunohistochemical evaluation. The expression or absence of markers is able to predict biological behavior and therapeutic respons $\mathrm{e}^{7,13,17}$. Among them, positivity for PR and ER favorably correlates with prognosis and hormonal therapeutic effect ${ }^{13}$, unlike HER2, which is usually associated with aggressiveness and hormonal resistance ${ }^{7,17}$.

Belonging to the family of tumors with TN immunophenotype, the combination of the absence of expression of endocrine receptors (ER and PR) and HER2 results in a favorable prognosis for patients with breast $\mathrm{AdCC}^{2,8,18}$. In the last decade, studies concluded that the neoplasm is well-located, especially in the retroareolar region, with a high survival rate of approximately $95 \%$ in 10 years and, in tumors measuring less than $14 \mathrm{~mm}$, there is no lymph node involvement ${ }^{2,16}$. Although uncommon, there are records of cases reporting axillary lymph node involvement, metastases to lungs, bones, livers, brain, and kidneys ${ }^{2,8,16}$, mainly observed in AdCCs with high-grade transformation, in which the glandular histological pattern is essentially replaced with a solid area, a subtype with worse prognosis ${ }^{2,4,9,12,18}$.

Breast $\mathrm{AdCC}$ is not restricted to the female population; there are epidemiological studies that show this rare neoplasm in men $^{2,18}$.A retrospective analysis of 19 cases of AdCC treated at a Canadian hospital reported involvement in a 53-year-old man, with a tumor measuring $4.0 \mathrm{~cm}$, lymph node involvement, and presence of metastasis ${ }^{18}$.

Although TN tumors have a clinical profile related to worse prognosis and resistance to hormonal therapy and trastuzumab, AdCC has an essentially favorable prognosis and can be conservatively treated ${ }^{2,14,15,18}$. To date, there is no consensus on the ideal treatment for $\mathrm{AdCC}^{8,10}$. Based on the characteristics of the tumor and the patient's immunological conditions, breast-conserving surgery, mastectomy, chemotherapy, or radiotherapy are indicated ${ }^{2,10}$. This adjuvant modality is prioritized when lymph node dissemination is detected ${ }^{10,16,18}$. In situations similar to that of the studied patient, the breast-conserving sectionectomy surgery with subsequent follow-up was adopted, considering the reduced size of the tumor and the absence of lymph node or hematogenous dissemination ${ }^{10,16}$.

\section{CONCLUSION}

Adenoid cystic carcinoma of the breast is part of the triple-negative tumor family and presents a paradoxically benign behavior when compared with its peers. As it is a rare tumor, the diagnosis can be facilitated through special histological techniques and the evaluation of the molecular or genomic profiling. Margin-free surgical excision is the standardized therapeutic approach, followed by clinical follow-up established between the mastologist and the patient. Although even rarer, there are records in the literature of recurrence and metastasis. Authors of the present article emphasize the importance of conducting further studies to elucidate the etiopathogenesis of breast AdCC, aiming to understand the natural history of this tumor and the mechanisms that allow it to behave differently.

\section{AUTHORS' CONTRIBUTIONS}

M.L.M.: conceptualization, data curation, investigation, methodology, project management, writing - original draft, writing review \& editing.

A.T.: resources, project administration, funding acquisition, data curation, methodology, writing - original draft, writing review \& editing.

A.A.L.L.: resources, conceptualization, methodology, supervision, writing - original draft, writing - review \& editing.

C.A.S.R.: resources, formal analysis, methodology, writing - original draft, writing - review \& editing.

L.C.A.: data curation, resources, writing - original draft, writing - review \& editing.

\section{REFERENCES}

1. Instituto Nacional de Câncer José Alencar Gomes da Silva. A situação do câncer de mama no Brasil: síntese de dados dos sistemas de informação [Internet]. Brazil: Instituto Nacional de Câncer José Alencar Gomes da Silva; 2019 [accessed on November 25, 2020]. Available from: https:/www.inca.gov.br/sites/ufu.sti.inca.local/ files/media/document/a_situacao_ca_mama_brasil_2019.pdf

2. World Health Organization(WHO).WHO Classification of Tumours Editorial Board. Breast tumours [Internet]. $5^{\mathrm{a}}$ ed. Lyon:International Agency for Research on Cancer; 2019 [accessed on November 24, 2020].v. 2. Available from: https://publications.iarc.fr/581
3. Brasil. Ministério da Saúde. Sistema de Informação do Colo do Útero. Sistema de Informação do câncer de mama. Datasus [Internet]. Ministério da Saúde [accessed on November 24, 2020]. Available from: http://w3.datasus.gov.br/siscam/index. php?area $=0402$

4. Vranic S, Bender R, Palazzo J, Gatalica Z. A review of adenoid cystic carcinoma of the breast with emphasis on its molecular and genetic characteristics. Hum Pathol. 2013;44(3):301-9. https://doi.org/10.1016/j. humpath.2012.01.002 
5. Goldblum JR, Lamps LW, McKenney JK, Myers JL. Rosai and Ackerman's Surgical Pathology. 9a ed. Philadelphia: Elsevier; 2004. v. 2. 1828 p.

6. Epstein J, Simpson J, Sanders M. Differential Diagnosis in Surgical Pathology: Breast. Philadelphia: Wolters Kluwer Health; 2016.

7. Wolff AC, Hammond MEH, Allison KH, Harvey BE, Mangu PB, Bartlett JMS, et al. Human Epidermal Growth Factor Receptor 2 Testing in Breast Cancer: American Society of Clinical Oncology/ College of American Pathologists Clinical Practice Guideline Focused Update. Arch Pathol Lab Med. 2018;142(11):1364-82. https://doi.org/10.5858/arpa.2018-0902-sa

8. Ghabach B, Anderson WF, Curtis RE, Huycke MM, Lavigne JA, Dores GM. Adenoid cystic carcinoma of the breast in the United States (1977 to 2006): a population-based cohort study. Breast Cancer Res. 2010;12:R54. https://doi.org/10.1186/bcr2613

9. Cambruzzi E, Pêgas KL, Zettler CG, Zettler EW. Carcinoma adenoide cístico de mama: relato de caso de uma rara neoplasia. Rev AMRIGS. 2012;56(2):161-3.

10. Romeira D, Cardoso D, Miranda H, Martins A. Adenoid cystic carcinoma: triple negative breast cancer with good prognosis. BMJ Case Rep. 2016;2016:bcr2015213704. https:// doi.org/10.1136/bcr-2015-213704

11. Ferlay J, Colombet M, Soerjomataram I, Matheus C, Parkin DM, Piñeros M, et al. Estimating the global cancer incidence and mortality in 2018: Globocan sources and methods. Int J Cancer. 2019;144(8):1941-53. https://doi.org/10.1002/ijc.31937
12. Righi A, Lenzi M, Morandi L, Flamminio F, De Biase D, Farnedi A, et al. Adenoid cystic carcinoma of the breast associated with invasive duct carcinoma: a case report. Int J Surg Pathol. 2011;19(2):230-4. https://doi.org/10.1177/1066896909332321

13. Allison KH, Hammond MEH, Dowsett M, McKernin SE, Carey LA, Fitzgibbons PL, et al. Estrogen and Progesterone Receptor Testing in Breast Cancer: ASCO/CAP Guideline Update. J Clin Oncol. 2020;38(12):1346-66. https://doi.org/10.1200/JCO.19.02309

14. Stoeck A, Lejnine S, Truong A, Pan L, Wang H, Zang C, et al. Discovery of biomarkers predictive of GSI response in triple-negative breast cancer and adenoid cystic carcinoma. Cancer Discov. 2014;4(10):1154-67.https://doi.org/10.1158/2159-8290.CD-13-0830

15. Yin L, Duan JJ, Bian XW, Yu SC. Triple-negative breast cancer molecular subtyping and treatment progress. Breast Cancer Res. 2020;22(1):61. https://doi.org/10.1186/s13058-020-01296-5

16. Thompson K, Grabowski J, Saltzstein SL, Sadler GR, Blair SL. Adenoid cystic breast carcinoma: is axillary staging necessary in all cases? Results from the California Cancer Registry. Breast J. 2011;17(5):485-9. https://doi.org/10.1111/j.1524-4741.2011.01117.x

17. VeeraraghavanJ,DeAngelis C,Reis-FilhoJS,PascualT,PratA,Rimawi MF, et al. De-escalation of treatment in HER2-positive breast cancer: Determinants of response and mechanisms of resistance. Breast. 2017;34(Suppl. 1):S19-S26. https://doi.org/10.1016/j.breast.2017.06.022

18. MillarBM,KerbaM,Youngson B,Lockwood GA,LiuF. The potential role of breast conservation surgery and adjuvant breast radiation for adenoid cystic carcinoma of the breast. Breast Cancer Res Treat. 2004;87:225-32. https://doi.org/10.1007/s10549-004-8693-z 